PROCEEDINGS OF THE

AMERICAN MATHEMATICAL SOCIETY

Volume 133, Number 6, Pages 1677-1683

S 0002-9939(04)07877-3

Article electronically published on December 21, 2004

\title{
MONOTONE PERIODIC ORBITS FOR TORUS HOMEOMORPHISMS
}

\author{
KAMLESH PARWANI
}

(Communicated by Michael Handel)

\begin{abstract}
Let $f$ be a homeomorphism of the torus isotopic to the identity and suppose that there exists a periodic orbit with a non-zero rotation vector $\left(\frac{p}{q}, \frac{r}{q}\right)$. Then $f$ has a topologically monotone periodic orbit with the same rotation vector.
\end{abstract}

\section{INTRODUCTION}

In this article we prove a theorem concerning the existence of topologically monotone periodic orbits on the torus. The concept of monotone orbits on the annulus is certainly not new; it goes back to Aubry and Mather's proof of the existence of orbits whose radial order is preserved by an area-preserving twist map of the annulus. These orbits that have their radial order preserved by the map are called Birkhoff orbits (see $\mathrm{Ka}$ ) or monotone orbits.

This notion of monotone orbits inspired the definition of topologically monotone orbits in Bd1, where Boyland proved that any homeomorphism of the annulus isotopic to the identity that has a periodic orbit with a non-zero rotation number $\frac{p}{q}$ also has a topologically monotone periodic orbit with the same rotation number. A topologically monotone periodic orbit has the property that the isotopy class of the map, keeping the periodic orbit fixed as a set, is of finite order. The main tool used in Boyland's proof is Nielsen-Thurston theory.

In [ML, Llibre and Mackay asked whether a similar result was true for torus homeomorphisms. The goal of this paper is to answer that question by proving the same theorem on the torus.

Main Theorem. If $f$ is a torus homeomorphism isotopic to the identity that has a periodic orbit with a non-zero rotation vector $\left(\frac{p}{q}, \frac{r}{q}\right)$, then falso has a topologically monotone periodic orbit with the same rotation vector.

There are some immediate complications one encounters while trying to generalize the theorem to the torus. First, the torus has rotation vectors instead of rotation numbers. Then on the annulus, under certain restrictions to the rotation number, we can only get the $p A$ (pseudoAnosov) isotopy class or the finite order isotopy class, but for the torus, there is the reducible isotopy class to deal with also.

Received by the editors January 12, 2004.

2000 Mathematics Subject Classification. Primary 37E30, 54H20; Secondary 58F20, 57M60.

Key words and phrases. Homeomorphisms, periodic orbits, rotation vectors.

(C) 2004 American Mathematical Society

Reverts to public domain 28 years from publication 
These concepts will be introduced in Section 1, and then in Section 2 we prove the main theorem of this paper.

It should be noted that LeCalvez has proved the existence of topologically monotone periodic orbits on the torus under the assumption that the maps are smooth by using variational techniques (see $[\mathrm{Lc}]$ ). Since we are dealing with homeomorphisms, we rely solely on topological methods.

\section{Definitions And important Results}

1.1. Rotation vectors. Let $f$ be a homeomorphism of the torus which is isotopic to the identity and let $F$ be its lift to the universal cover $\widetilde{T^{2}}$, the plane. Let ()$_{1}$ and ()$_{2}$ be the projections of a point in the plane to the $x$-axis and the $y$-axis respectively and let $x$ be a point on the torus $T^{2}$ with $\widetilde{x}$ as its lift. Then the rotation vector of $\widetilde{x}$, with respect to a lift $F$, is defined as follows if the limit exists:

$$
\rho(\widetilde{x}, F)=\left(\lim _{n \rightarrow \infty}\left(\frac{F^{n}(\widetilde{x})-\widetilde{x}}{n}\right)_{1}, \lim _{n \rightarrow \infty}\left(\frac{F^{n}(\widetilde{x})-\widetilde{x}}{n}\right)_{2}\right) .
$$

If $x$ is a periodic point, say of period $q$, then the rotation vector is always well defined and can be written as $\left(\frac{p}{q}, \frac{r}{q}\right)$ for some integers $p$ and $r$. In fact, the rotation vector for any point on the orbit of $x$ is the same, and so, we can associate the vector $\left(\frac{p}{q}, \frac{r}{q}\right)$ to the periodic orbit. Periodic orbits with rotation vector $\left(\frac{p}{q}, \frac{r}{q}\right)$ and least period $q$ will be called $(p, r, q)$ orbits. Note that a $(p, r, q)$ orbit has the same rotation vector as a $(p t, r t, q t)$ orbit, where $t$ is some positive integer.

The covering space of the torus comes naturally equipped with two important covering translations. Define $X(\widetilde{x})=\widetilde{x}+(1,0)$ and $Y(\widetilde{x})=\widetilde{x}+(0,1)$. Clearly, the rotation vector of a point depends on the lift, and the relationship is $\rho\left(\widetilde{x}, Y^{m} X^{n} F\right)=\rho(\widetilde{x}, F)+(n, m)$, where $n$ and $m$ are integers. So when we discuss periodic orbits with a certain rotation vector in this paper, we assume the existence of some lift for which that rotation vector is realized. Also, when we start with a periodic orbit, say the $(p, r, q)$ orbit, and then prove that another $(p, r, q)$ orbit exists, it is to be understood that both rotation vectors are calculated with respect to the same lift.

1.2. The Nielsen-Thurston Classification Theorem and braids. Every orientation-preserving homeomorphism of an orientable surface with negative Euler characteristic is isotopic to a homeomorphism $g$ such that either

a) $g$ is finite order, or

b) $g$ is pseudoAnosov $(p A)$, or

c) $g$ is reducible.

A map $g$ is said to be reducible if there is a disjoint collection $C$ of non-parallel, non-peripheral simple disjoint curves such that $g$ leaves invariant the union of disjoint regular neighborhoods of curves in $C$, and the first return map on each complementary component is either of finite order or $p A$.

This classification theorem was first announced in $\mathrm{Th}$, and the proofs appeared later in $[\overline{F L P}$ and $[\mathrm{CB}]$.

The torus doesn't have negative Euler characteristic but, following Handel as in $[\mathrm{Hn}]$, we will examine the isotopy class relative to a periodic orbit; this will introduce punctures and provide the negative Euler characteristic to apply the Nielsen-Thurston Classification Theorem. When the isotopy class relative to a given periodic orbit is of finite order, the periodic orbit is called a finite order periodic 
orbit, and reducible and $p A$ periodic orbits are defined similarly. The isotopy class relative to a periodic orbit is also referred to as the braid of the periodic orbit.

Definition 1.1. Let $x$ and $y$ be two distinct periodic points of least period $n$ for homeomorphisms $f$ and $g$ respectively of the same orientable surface $S$. Then the orbit of $x(O(x))$ and the orbit of $y(O(y))$ have the same braid if there exists an orientation-preserving homeomorphism $h$ of $S$ with the property that $h$ maps $O(x)$ onto $O(y)$ and the isotopy class of $h^{-1} f h$ relative to the orbit of $y$ is the same as the isotopy class of $g$ relative to the orbit of $y$, that is, $\left[h^{-1} f h\right]_{O(y)}=[f]_{O(y)}$.

A periodic orbit has a trivial braid if the isotopy class relative to the periodic orbit is of finite order, that is, there exists a homeomorphism $g$ isotopic to $f$, relative to the periodic orbit, such that $g^{n}=i d e n t i t y$ for some $n$. In other words, finite order periodic orbits have trivial braids. These periodic orbits are considered to be topologically monotone.

A periodic orbit has a non-trivial braid if the isotopy class relative to the periodic orbit is not of finite order. In other words, periodic orbits with non-trivial braids are either reducible periodic orbits or are $p A$ periodic orbits. These periodic orbits are not topologically monotone.

Boyland defined a natural partial order $(\triangleright)$ into these braids. If $\alpha$ and $\beta$ are two braids of periodic orbits, then $\alpha \triangleright \beta$ if and only if the existence of a periodic orbit with braid $\alpha$ in any homeomorphism $f$ on a given surface implies the existence of a periodic orbit with braid $\beta$ for the same $f$. The proof of the fact that this is an actual partial order is not easy and is in $\mathrm{Bd} 1$. He also proved that a $p A$ periodic orbit is strictly above (in the partial ordering) all other periodic orbits that are present in the $p A$ representative of the isotopy class relative to the $p A$ periodic orbit.

The existence of topologically monotone periodic orbits on the annulus in Bd1] is established by showing that periodic orbits with non-trivial braids force the existence of periodic orbits with trivial braids and the same rotation number (nontrivial $\triangleright$ trivial).

Theorem 1.2 (Boyland). Let $f$ be a homeomorphism of the annulus isotopic to the identity. If $f$ has a periodic orbit with non-zero rotation number $\frac{p}{q}$, then $f$ also has a topologically monotone periodic orbit with the same rotation number.

Essentially, we follow the same strategy on the torus, and the proof of the main theorem in Section 2 is broken into two parts, reducible $\triangleright$ finite order and $p A \triangleright$ finite order.

We will also need the following result, which can be obtained from the arguments in [BHG] and is also proved in [Pa2].

Theorem 1.3. Let $f$ be a homeomorphism of the torus that is isotopic to the identity and has a $p A$ periodic orbit. Let $g$ be the $p A$ representative of the isotopy class relative to this orbit and let $G$ be its lift to the plane that fixes the lifts of all the points in the $p A$ orbit. Then $G$ has a dense orbit.

We will use this theorem in the next section to prove $p A \triangleright$ trivial. 


\section{FINITE ORDER PERIODIC ORBITS ON THE TORUS}

In this section we prove the main theorem by showing that finite order periodic orbits are on the bottom in the partial ordering of periodic orbits for torus homeomorphisms isotopic to the identity, that is, the reducible $\triangleright$ finite order and $p A \triangleright$ finite order. We restrict our attention to periodic orbits with the same non-zero rotation vector, say $\left(\frac{p}{q}, \frac{r}{q}\right)$, and assume that there are no common factors between $p, r$, and $q$. Later we reduce the general case, in which there may be a common factor between $p, r$, and $q$, to the case of no factors.

Theorem 2.1. Let $f: T^{2} \rightarrow T^{2}$ be a homeomorphism isotopic to the identity. Suppose there exists a $(p, r, q)$ orbit such that $\operatorname{gcd}(p, r, q)=1$. Then there exists a finite order $(p, r, q)$ orbit.

Proof. If the given periodic orbit is already of finite order type, then we're done. If not, the proof breaks down into two cases - the periodic orbit is either of reducible type or it is of $p A$ type. These are handled separately below.

Reducible case. We assume that we obtain a reducible isotopy class, keeping the periodic orbit fixed. A reducing curve can be of two types - essential or nonessential (this is with respect to the unpunctured torus). A reducing curve cannot be non-essential for this would imply that there is a common factor between $p, r$, and $q$. In fact, the common factor would be exactly the number of punctures contained in the disc bounded by the non-essential curve.

So any reducing curve must be essential. All the essential reducing curves are disjoint, and thus, split the torus into parallel annuli. These annuli have an equal number of punctures, say $n$ punctures, in their interiors and are permuted by the action of the map $g$, which represents this reducible isotopy class.

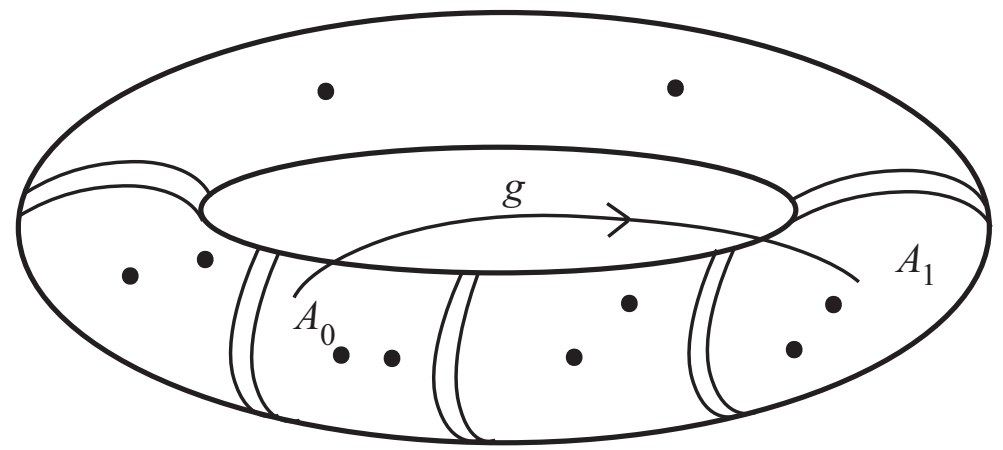

Figure 1. The Reducible Case

In this case, we will show that we can obtain a finite order isotopy class, keeping a periodic orbit with the same period and rotation vector (it may not be the same periodic orbit that we start with). Let $A_{0}, A_{1}, A_{2}, \ldots, A_{m-1}$ be the annuli that $g$ permutes, numbered so that $g\left(A_{k}\right)=A_{k+1} \bmod m$ and $n m=q$.

CASE 1. Suppose that the maps $g^{m}: A_{k} \rightarrow A_{k}$ relative to the punctures are all of finite order. It is easy to see that all these maps are conjugate to each other, and so if one is of finite order, then all are of finite order. Since all finite order maps on 
the annulus are conjugate to rotations, it follows that $g^{m n}\left(\right.$ or $\left.g^{q}\right)$ is the identity in each annulus.

We will now argue that this implies that $g^{q}$ is isotopic to the identity on the entire torus relative to the $(p, r, q)$ orbit. The complements of the interiors of annuli containing the punctures (the $A_{k}$ 's) are closed annuli that do not contain any punctures. Observe that $g^{q}$ fixes the boundary components of these unpunctured annuli since it fixes the boundary components of the $A_{k}$ 's. So if $g^{q}$ is isotopic to the identity in all these unpunctured annuli relative to their boundaries (keeping the boundaries fixed throughout the isotopy), then $g^{q}$ is isotopic to the identity on the entire torus relative to the $(p, r, q)$ orbit, because we already know that $g^{q}$ is the identity on the annuli containing the punctures. Now suppose $g^{q}$ is not isotopic to the identity in one of these unpunctured annuli relative to its boundary components. Then $g^{q}$ must be isotopic to some non-trivial Dehn twists. It is easy to see that the maps on all these annuli are all conjugate to each other, so $g^{q}$ is isotopic to the same Dehn twists in each unpunctured annulus. However, $g^{q}$ is isotopic to the identity on the entire torus when the punctures are allowed to move, because $g$ is isotopic to $f$ and $f$ is isotopic to the identity by assumption. If we have non-trivial Dehn twists that don't cancel each other out (because they are identical), then $g^{q}$ is not isotopic to the identity, which is a contradiction. It follows that $g^{q}$ is isotopic to the identity on the entire torus relative to the $(p, r, q)$ orbit.

To show that the $(p, r, q)$ orbit is topologically monotone, we require a map isotopic to $g$ relative to the orbit that is of finite order. Such a map is guaranteed by Fenchel's solution to the Nielsen Realization problem for finite solvable groups (see Chapter 3 in $[\mathrm{Zg}]$ ), which provides a map $h$ isotopic to $g$, relative to the $(p, r, q)$ orbit, such that $h^{q}$ is the identity. This shows that the $(p, r, q)$ orbit is of finite order type, that is, it is topologically monotone.

CASE 2. Suppose that the maps $g^{m}: A_{k} \rightarrow A_{k}$ relative to the punctures are all $p A$. It now follows from Boyland's proof of Theorem 1.2 in [Bd1] that there exists a finite order periodic orbit with the same period and rotation number in each $A_{k}$. Since all these annulus maps are conjugate, the periodic orbits connect in the torus to give a periodic orbit with the same rotation vector and period as the originally punctured orbit. This reduces to Case 1, and we can find an isotopy relative to the new orbit such that the isotoped map is of finite order. Because the periodic orbits in the $p A$ components are unremovable (see $[\mathrm{Bd} 2]$ ), this periodic orbit existed in the original map $f$.

We have actually established a stronger result. If we do obtain a finite order periodic orbit which is distinct from the one we began with, then it is strictly below the original orbit in the partial order. This is because the only way for the original orbit to not be of a finite order type is for the reducible components to be $p A$, that is, the maps $g^{m}: A_{k} \rightarrow A_{k}$ are $p A$. Also, $p A$ orbits are strictly above all periodic orbits that are present in the $p A$ representative of the isotopy class (see Bd1).

$\boldsymbol{p A}$ case. In Lemma 2.3, we will prove that any $p A(p, r, q)$ orbit forces another $(p, r, q)$ orbit. Boyland proved that this other periodic orbit is strictly below the $p A$ orbit in the partial order (see $[\mathrm{Bd} 1])$. Furthermore, there are only finitely many periodic orbits of any given period in any $p A$ map. So consider a minimal $(p, r, q)$ orbit in the partial order. If it's $p A$, there is another $(p, r, q)$ orbit below it-so it's not minimal. If it's reducible and not of finite order, then it forces another 
$(p, r, q)$ finite order orbit (by the argument above for the reducible case). Thus, any minimal $(p, r, q)$ orbit must be of finite order and there is at least one minimal orbit.

It now remains to prove Lemma 2.3. We shall appeal to the following result in Fa for the existence of a fixed point of positive index. The proof is based on the ideas used to demonstrate the Brouwer Plane Translation Theorem (see $[\mathrm{Fr}]$ ).

Theorem 2.2 (Fathi). Let $G: R^{2} \rightarrow R^{2}$ be an orientation-preserving homeomorphism which possesses a non-wandering point. Then $G$ has a fixed point. If $G$ has only isolated fixed points, then it has a fixed point of positive index.

Lemma 2.3. Let $g: T^{2} \rightarrow T^{2}$ be the $p A$ representative obtained from $f$, keeping the $(p, r, q)$ orbit fixed throughout the isotopy. Then $g$ has another $(p, r, q)$ periodic orbit.

Proof. Let $g$ be the $p A$ representative obtained relative to the $(p, r, q)$ orbit. Let $G$ be the lift to the plane that realizes the rotation vector $\left(\frac{p}{q}, \frac{r}{q}\right)$ for the $p A$ orbit and then consider $X^{-p} Y^{-r} G^{q}$; call this map $H$. Then by Theorem 1.3, we know that $H$ has a dense orbit and so there is a non-wandering point. Since the periodic points (of any given period) are isolated in a $p A$ map (see [FLP]), the fixed points of $H$ on the plane are also isolated. So, by Theorem 2.2, we have a fixed point with positive index for $H$, which is a $(p, r, q)$ orbit for $g$, and each point in the orbit is fixed with positive index for $g^{q}$.

The $p A(p, r, q)$ orbit is the location of all the one-prongs or needles, and these needles have index zero. Since there is a fixed point for $g^{q}$ with positive index and because the indices have to add up to zero, we have actually shown that the $p A$ periodic orbit forces at least two other $(p, r, q)$ periodic orbits.

Proof of the main theorem. Let $f$ be a homeomorphism of the torus isotopic to the identity and suppose there exists a periodic orbit with rotation vector $\left(\frac{p}{q}, \frac{r}{q}\right)$. Also assume that this is a $(p t, r t, q t)$ periodic orbit, where $t$ is a positive integer and there are no common factors between $p, r$, and $q$. Let $F$ be the lift to the plane and consider $X^{-p} Y^{-r} F^{q}$. We obtain a periodic point of period $t$ for $X^{-p} Y^{-r} F^{q}$. Then by Theorem 2.2, we also obtain a fixed point for $X^{-p} Y^{-r} F^{q}$, which has period $q$ for $f$ and rotation vector $\left(\frac{p}{q}, \frac{r}{q}\right)$, that is, it is a $(p, r, q)$ orbit. Thus, without loss of generality, we may assume that we have a $(p, r, q)$ orbit where there are no common factors between $p, r$, and $q$. Now, by Theorem 2.1, we obtain a $(p, r, q)$ orbit that is topologically monotone and it has the desired rotation vector $\left(\frac{p}{q}, \frac{r}{q}\right)$.

It is natural to ask if there is a similar theorem for non-periodic orbits with irrational rotation vectors. This question is unanswered even for the annulus. A similar theorem about the existence of monotone periodic orbits has been proved for periodic orbits on surfaces of higher genus (see [Pa1]).

\section{ACKNOWLEDGMENTS}

The author would like to thank John Franks and Philip Boyland for several useful and stimulating conversations. 


\section{REFERENCES}

[Bd1] P. Boyland, Rotation sets and monotone orbits for annular homeomorphisms, Comm. Math. Helv., 67 (1992), 203-213. MF.1161281 (93k:58180)

[Bd2] P. Boyland, Isotopy stability for dynamical systems on surfaces, Geometry and topology in dynamics, Contemp. Math., 246 (1999), 17-45. MR.1732369 (2001i:37063)

[BHG] P. Boyland, T. Hall, and J. Guaschi, L'ensemble de rotation des homéomorphismes pseudo-Anosov, C.R. Acad. Sci. Paris Sér. I Math., 316 (1993), 1077-1080. MR1222976 (94g:58175)

[CB] A. Casson, S. Bleiler, Automorphisms of surfaces after Nielsen and Thurston, London Math. Soc., Stud. Texts, 9 (1988), Cambridge University Press. MR0964685 (89k:57025)

[Fa] A. Fathi, An orbit closing proof of Brouwer's lemma on translation arcs, Ensign. Math., 33 (1987), 315-322. MR0925994 (89d:55004)

[FLP] A. Fathi, F. Laudenbach, V. Poenaru, Travaux de Thurston sur les surfaces, Asterisque 66-67 (1979). MF0568308 (82m:57003)

[Fr] J. Franks, Generalizations of the Poincaré-Birkhoff theorem, Ann. of Math., 128 (1988), 139-151. MR 0951509 (89m:54052)

[Hn] M. Handel, The rotation set of a homeomorphism of the annulus is closed, Comm. Math. Phys., 127 (1990), no. 2, 339-349. MR1037109 (91a:58102)

[Ka] A. Katok, Some remarks on the Birkhoff and Mather twist theorems, Ergod. Th. and Dynam. Sys., 2 (1982), 183-194. MR0693974 (84m:58041)

[Lc] P. LeCalvez, Dynamical properties of diffeomorphisms of the annulus and of the torus, SMF/AMS Texts and Monographs 4, American Mathematical Society, Providence (2000). MR1747730 (2000m:37059)

[ML] J. Llibre and R. Mackay, Rotation vectors and entropy for homeomorphisms of the torus isotopic to the identity, Ergodic Theory Dynam. Systems 11 (1991), no. 1, 115-128. MR1101087 (92b:58184)

[Pa1] K. Parwani, Simple braids for surface homeomorphisms, preprint.

[Pa2] K. Parwani, Thesis, Northwestern University, 2003.

[Th] W. Thurston, On the geometry and dynamics of diffeomorphisms of surfaces, Bull. A.M.S., 19 (1988), 417-431. MR0956596 (89k:57023)

[Zg] H. Zieschang, Finite groups of mapping classes of surfaces, Lecture Notes in Mathematics 875. Springer-Verlag, Berlin (1981). MR0643627|(86g:57001)

Department of Mathematics, Northwestern University, Evanston, Illinois 60208

E-mail address: forty2@math.northwestern.edu 